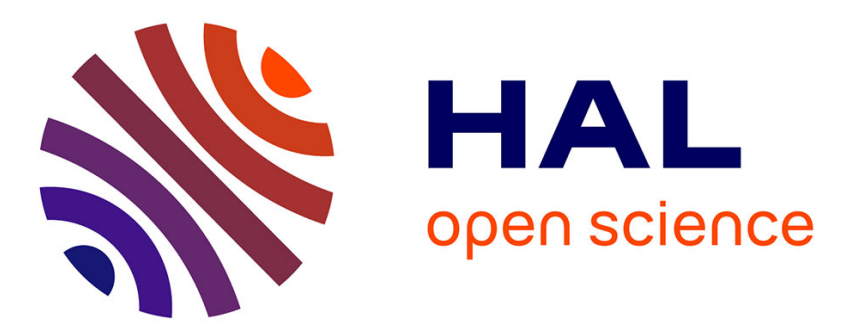

\title{
A Collaborative Observer for Switched Linear Systems with Unknown Inputs
}

Jorge Dávila, Tarek Raissi, Xubin Ping

\section{To cite this version:}

Jorge Dávila, Tarek Raissi, Xubin Ping. A Collaborative Observer for Switched Linear Systems with Unknown Inputs. 2021 American Control Conference, May 2021, New Orleans, United States. hal03226024

\section{HAL Id: hal-03226024 \\ https://hal.science/hal-03226024}

Submitted on 13 May 2021

HAL is a multi-disciplinary open access archive for the deposit and dissemination of scientific research documents, whether they are published or not. The documents may come from teaching and research institutions in France or abroad, or from public or private research centers.
L'archive ouverte pluridisciplinaire HAL, est destinée au dépôt et à la diffusion de documents scientifiques de niveau recherche, publiés ou non, émanant des établissements d'enseignement et de recherche français ou étrangers, des laboratoires publics ou privés. 


\title{
A Collaborative Observer for Switched Linear Systems with Unknown Inputs
}

\author{
Jorge Dávila, Senior Member, IEEE, Tarek Raïssi, Senior Member, IEEE, Xubin Ping
}

\begin{abstract}
In this article, a collaborative observer is proposed for a class of Linear Switched Systems affected by unknown inputs. The class of switched systems under study accepts a dwell-time and has a completely known exogenous switching signal. Two main elements compose the proposed observer: a point observer designed using High-Order Sliding-Modes, which provides finite-time exact convergence in the presence of disturbances with a time-varying known upper bound, and a collaborative observer that gives an interval estimation of the state in the presence of the unknown inputs.
\end{abstract}

\section{INTRODUCTION}

Switched systems consist of a finite number of operation modes and a "switching logic" that sets the current mode of operation through instantaneous transitions from one mode to another. Thus, in these systems, a discrete and continuous state can be distinguished. The first one, which corresponds to the active operation mode, is an integer number. The second corresponds to the set of continuous variables that evolve in time, according to the active mode's correspondent dynamic. The stability of this class of systems has been studied using common Lyapunov functions [1], as well as introducing the concept of dwell-time to prove the convergence of the systems using the time between switchings [2]. The reconstruction of the continuous and/or discrete states by means of output measurements is actively studied [3]. However, state estimation in the presence of multiple unknown inputs appears to be of particular challenge.

Related to the observation problem for switched systems, i.e., the continuous and discrete state estimation, the observer design is of great interest for many control areas. In [4] and [5], a Luenberger observer approach is combined with a high-order sliding-mode observer for linear systems are proposed for the known operating mode case. In [6], a hybrid system subject to input disturbances is considered, and an algorithm based on the moving horizon estimation method is applied for simultaneous state and input estimation. Considering that the continuous state is known, an algorithm for reconstructing the discrete state in nonlinear uncertain switched systems is presented in [7] based on sliding-mode control theory. On the other hand, for the unknown operating mode case, in [8], based on a property of strong detectability

Jorge Dávila is with the Instituto Politécnico Nacional, Section of Graduate Studies and Research, ESIME -Ticomán, Mexico City, Mexico, e-mail: jadavila@ieee.org.

Tarek Raïssi is with the Conservatoire National des Arts et Metiers (CNAM), Cedric - lab 292, Rue Saint-Martin, 75141 Paris Cedex 03, France, e-mail: tarek.raissi@cnam.fr.

Xubin Ping is with the Department of Automation, School of ElectroMechanical Engineering, Xidian University, Xi'an, 710075, China, e-mail: pingxubin@126.com. and using an LMI approach, are designed two-state observers for some classes of switched linear systems with unknown inputs. In [9], considering that the output and an initial state are available, a necessary and sufficient condition for a switched system's invertibility is proposed. In the same context, a nonlinear finite time observer to estimate the capacitor voltage for multicellular converters, which have a switched behavior, is proposed by [10]. In [11], based on the nonhomogeneous high-order sliding mode approach, a robust observer for the unknown and exogenous switching signal is proposed to solve the problem of continuous and discrete state estimation for a class of nonlinear switched systems. In [12], a robust observer designed using high-order sliding-modes is proposed to reconstruct in finite-time the state of Switched Linear Systems that satisfies the strong observability conditions.

Several works have investigated estimation problems, using the interval observers for different classes of timeinvariant and parameter-varying systems ([13], [14], [15], [16], [17]). Furthermore, interval unknown input observers have received great attention. See, for instance [18], [19]. Using the "relative degree" property, unknown input observers decouples the unknown input from the state vector.

This work extends the class of disturbances supported for high-order sliding-mode based observers for switched linear systems, like the one presented in [12], allowing the estimation of systems subject to disturbances with timevarying upper bounds. Besides this, the proposed observer also provides an interval estimation of the state in the presence of a broader class of perturbations than the allowed by [20], and guarantees an interval estimation of the state even in the presence of unknown inputs with an unknown relative degree concerning the system's output.

\section{Problem statement}

Consider the following switched linear system with unknown inputs:

$$
\begin{aligned}
\dot{x}(t) & =A_{\lambda(t)} x(t)+B_{\lambda(t)} u(t)+D_{\lambda(t)} d(t)+E_{\lambda(t)} w(t)(1) \\
y(t) & =C_{\lambda(t)} x(t)
\end{aligned}
$$

where $x(t) \in \mathbb{R}^{n}, u(t) \in \mathbb{R}^{m}, y(t) \in \mathbb{R}^{p}, d(t) \in$ $\mathbb{R}^{q_{1}}, w(t) \in \mathbb{R}^{q_{2}}$ are the continuous state, the input, the output, the disturbances and the unknown input vectors, respectively. The discrete state is defined by $\lambda(t)$, which is a piecewise constant function that takes its values from an index set $\mathcal{I}=\overline{1, N}$, i.e. $\lambda(t): \mathbb{R}^{+} \rightarrow \mathcal{I}$. We say that the system is operating in the mode $i$ at time $t$ if $\lambda(t)=i$, 
to which it corresponds to the system a set of constant matrices $\left\{A_{i}, B_{i}, C_{i}, D_{i}, E_{i}\right\}$. Each set of constant matrices $A_{i}, B_{i}, C_{i}, D_{i}, i=\overline{1, N}$ is conformable and known. The distribution matrix $E_{i}$ is considered unknown but bounded by a constant value, i.e., $\left\|E_{i}\right\| \leq \overline{E_{i}}, i=\overline{1, N}$.

Let the disturbance and the unknown inputs be bounded by a known positive constant values $\bar{w}$ and a known timedependent function $\bar{d}(t)$, i.e. $\|w(t)\| \leq \bar{w},\|d(t)\| \leq \bar{d}(t)$, respectively. For constant values $\underline{x}, \bar{x}$ the state satisfies

$$
\underline{x} \preccurlyeq \quad x(t) \preccurlyeq \bar{x}
$$

The goal of this article is to design an interval observer for the switched system (1), (2) that provides a collaborative estimation of the continuous state $x(t)$ even in the presence of the disturbances $d(t)$ and the unknown inputs $w(t)$, which have an unknown relative degree with respect to the outputs.

Let $\lceil x\rfloor^{\alpha}=|x|^{\alpha} \operatorname{sign} x$ for the generic scalar argument $x$, in particular $\lceil x\rfloor^{0}=\operatorname{sign} x$. The sequence of integers $\{1,2,3, \ldots, N\}$ is denoted by $\overline{1, N}$. Let the precedence operator $\preccurlyeq / \prec$ be used to denote that all the elements of a vector or a matrix satisfy a specific inequality, i.e. for a matrix $M \in \mathbb{R}^{m \times n}$, the operation $(0 \preccurlyeq M / 0 \prec M)$ denotes that $\left(\left(0 \leq M_{i j}\right) /\left(0<M_{i j}\right)\right) \forall i=\overline{1, m}, j=\overline{1, n}$. For a matrix $N \in \mathbb{R}^{m \times n}$, let us define $N^{+}=\max \{0, N\}$, $N^{-}=N^{+}-N$ and $|N|=N^{+}+N^{-}$.

\section{PRELIMINARY CONCEPTS}

Let consider that the linear system evolves in the operation mode $\lambda(t)=\lambda, \lambda \in \mathcal{I}$, i.e., the system is represented by the specific set of matrices $\left\{A_{\lambda}, B_{\lambda}, C_{\lambda}, D_{\lambda}, E_{\lambda}\right\}$.

The Rosenbrock matrix of system (1), (2) in the mode $\lambda(t)=\lambda$ is defined as

$$
R_{\lambda}(s)=\left[\begin{array}{cc}
s I-A_{\lambda} & -D_{\lambda} \\
C_{\lambda} & 0
\end{array}\right]
$$

The invariant zeros of the system (1), (2) in the mode $\lambda$ are all the values $s_{i} \in \mathbb{C}$ for which $\operatorname{rank}\left(R_{\lambda}\left(s_{i}\right)\right)<$ $n+\operatorname{rank}\left(D_{\lambda}\right)$. Let's define $\omega\left(s_{i}\right)=\left\{x \mid x \in \operatorname{ker}\left(C_{\lambda}\right) \cap\right.$ $\left.\left(s_{i} I-A_{\lambda}\right) x \in \mathcal{R}\left(D_{\lambda}\right)\right\}$, where $\mathcal{R}\left(D_{\lambda}\right)$ denotes the range space of $D_{\lambda}$, then the dimension of the null space of (4) is given by $\operatorname{rank}\left(\cup_{\forall i} \omega\left(s_{i}\right)\right)=n_{\mathcal{V}}$.

The system is called strongly detectable [21] in the operation mode $\lambda$ if the invariant zeros of (1), (2) have strictly negative real parts, i.e. $\Re\left(s_{0}\right)<0 \forall s_{0} \in \mathbb{C} \mid \operatorname{rank} R_{\lambda}\left(s_{0}\right)<$ $n+\operatorname{rank}\left(D_{\lambda}\right)$.

Definition 1: The output vector $y=C_{\lambda} x$ is said to have a full vector relative degree $r_{\lambda}=\left(r_{1, \lambda}, \ldots, r_{p, \lambda}\right)$ with respect to the disturbance $d(t)$, if the matrix $C_{\lambda}$ is such that it satisfies the following equalities

$$
\begin{aligned}
c_{i, \lambda} A_{\lambda}^{j} D_{\lambda} & =0, \quad i=1, \ldots, p ; j=0, \ldots, r_{i}-2,(5) \\
c_{i, \lambda} A_{\lambda}^{r_{i}-1} D_{\lambda} & \neq 0
\end{aligned}
$$

and

$$
Q_{\lambda}=\left[\begin{array}{c}
c_{1, \lambda} A_{\lambda}^{r_{1}-1} D_{\lambda} \\
\vdots \\
c_{p, \lambda} A_{\lambda}^{r_{p}-1} D_{\lambda}
\end{array}\right], \quad \operatorname{rank} Q_{\lambda}=q_{1}
$$

where $Q_{\lambda} \in \mathbb{R}^{p \times q}$.

Lemma 1: [22] Let $\delta>0$ be a scalar and $P \in \mathbb{R}^{n \times n}$ be a symmetric positive definite matrix, then $2 x^{T} y \leq \frac{1}{\delta} x^{T} P x+$ $\delta y^{T} P^{-1} y$ for every $x, y \in \mathbb{R}^{n}$.

Lemma 2: [18] Let $x \in \mathbb{R}^{n}$ be a vector satisfying $\underline{x} \leq$ $x \leq \bar{x}$, and a constant matrix $M \in \mathbb{R}^{m \times n}$, then $M^{+} \underline{x}-$ $M^{-} \bar{x} \preccurlyeq M x \preccurlyeq M^{+} \bar{x}-M^{-} \underline{x}$.

Definition 2: [23] A matrix $A=\left(a_{i, j}\right) \in \mathbb{R}^{n \times n}$ is said to be Metzler if all its off-diagonal elements are nonnegative i.e. $a_{i, j} \geq 0, \forall(i, j), i \neq j$. It is said to be nonnegative if all the entries are nonnegative: $A \geq 0$.

Lemma 3: [24] The system described by

$$
\dot{x}(t)=A x(t)+u(t), x(0)=x_{0}
$$

is said to be nonnegative (or positive) if $A$ is a Metzler matrix and $u(t) \geq 0$. For any initial condition $x_{0} \geq 0$ the solution of (8) satisfies $x(t) \geq 0, \forall t \geq 0$.

\section{OBSERVER DESIGN}

\section{A. System transformation}

Assumption 1: For each operation mode $\lambda, \lambda=\overline{1, N}$, the system (1), (2) is observable.

Assumption 2: For each operation mode $\lambda, \lambda=\overline{1, N}$, the system (1), (2) has full vector relative degree with respect to the disturbance $d(t)$.

If Assumption 2 holds, there exists at least one combination of $q_{1}$ linearly independent rows of the matrix $Q_{\lambda}$ such that we can form a new matrix $Q_{\lambda}^{*} \in \mathbb{R}^{q_{1} \times q_{1}}$ satisfying:

$$
\operatorname{rank} Q_{\lambda}^{*}=q_{1}, \quad Q_{\lambda}^{*}=\left[\begin{array}{c}
c_{1, \lambda *} A_{\lambda}^{r_{1}-1} D_{\lambda} \\
\vdots \\
c_{1, \lambda *} A_{\lambda}^{r_{q_{1}}-1} D_{\lambda}
\end{array}\right]
$$

Then, it is possible to define the matrix

$O_{\lambda}=\left[\begin{array}{lll}\left(c_{1, \lambda *}\right)^{T} & \ldots\left(c_{1, \lambda *} A_{\lambda}^{r_{1}-2}\right)^{T} \quad \ldots\left(c_{q_{1}, \lambda *} A_{\lambda}^{r_{q_{1}}-2}\right)^{T}\end{array}\right]^{T}$,

built with the same rows of the matrix $C_{\lambda}$ that were used to form $Q_{\lambda}^{*}$, such that $\operatorname{rank} O_{\lambda}=n_{\mathcal{V}_{\lambda}}$.

Lemma 4: [25] Let the system (1), (2) be in the operation mode $\lambda$, and the output $y$ of (2) has the vector relative degree $r_{\lambda *}=\left(r_{1, \lambda *}, \ldots, r_{q_{1}, \lambda *}\right)$ with respect to the unknown input $d(t)$. Then the vectors $c_{1, \lambda *}, \ldots, c_{1, \lambda *} A^{r_{1 *}-1}, \ldots, c_{q, \lambda *}, \ldots, c_{q, \lambda *} A^{r_{q *}-1}$ are linearly independent.

Assumption 3: For each operation mode $\lambda, \lambda=\overline{1, N}$, the unknown inputs distribution matrices $E_{\lambda}$ satisfy the following equality $O_{\lambda} E_{\lambda}=0, \quad \lambda=\overline{1, N}$.

Assumption 3 allows that the unknown inputs can be matched with the disturbances.

Define the matrix $V_{\lambda}$ as the orthogonal complement to the matrix $\left[O_{\lambda}^{T}\right]$ (a matrix with $n-n_{\mathcal{V}_{\lambda}}$ linearly independent rows), and let $V_{\lambda}$ be selected such that $V_{\lambda} D_{\lambda}=0$. As a consequence of the selection of the matrices $O_{\lambda}$ and $V_{\lambda}$, the next $n \times n$ transformation matrix has full row rank:

$$
M_{\lambda}=\left[\begin{array}{c}
O_{\lambda} \\
V_{\lambda}
\end{array}\right]
$$


There exist two sets, one corresponding to the state transformations $\left\{M_{1}, M_{2}, \ldots, M_{N}\right\}$ and another of output transformations $\left\{S_{1}, S_{2}, \ldots, S_{N}\right\}$, each one suitable for its corresponding operation mode, such that the transformed state and the transformed output:

$$
\begin{aligned}
& \chi=M_{\lambda} x, \\
& y_{z}=S_{\lambda} y=S_{\lambda} C_{\lambda} M_{\lambda}^{-1} \chi
\end{aligned}
$$

for all $\lambda=\overline{1, N}$, takes the following form:

$$
\begin{aligned}
{\left[\begin{array}{l}
\dot{\chi}_{1} \\
\dot{\chi}_{2}
\end{array}\right]=} & {\left[\begin{array}{ll}
A_{11_{\lambda}} & 0 \\
A_{21_{\lambda}} & A_{22_{\lambda}}
\end{array}\right]\left[\begin{array}{l}
\chi_{1} \\
\chi_{2}
\end{array}\right]+\left[\begin{array}{l}
B_{1_{\lambda}} \\
B_{2_{\lambda}}
\end{array}\right] u(t) } \\
& +\left[\begin{array}{c}
D_{1_{\lambda}} \\
0
\end{array}\right] d(t)+\left[\begin{array}{l}
E_{1_{\lambda}} \\
E_{2_{\lambda}}
\end{array}\right] w(t) \\
{\left[\begin{array}{l}
y_{1} \\
y_{2}
\end{array}\right]=} & {\left[\begin{array}{cc}
C_{11_{\lambda}} & 0 \\
0 & C_{22_{\lambda}}
\end{array}\right]\left[\begin{array}{l}
\chi_{1} \\
\chi_{2}
\end{array}\right] }
\end{aligned}
$$

with $\chi_{1} \in \mathbb{R}^{n_{\mathcal{V}_{\lambda}}}, \chi_{2} \in \mathbb{R}^{\left(n-n_{\mathcal{V}_{\lambda}}-n_{\mathcal{N}_{\lambda}}\right)}$. Assumption 3 allows to characterize the form of matrix $E_{1_{\lambda}}$. The matrices $A_{11_{\lambda}}, D_{1_{\lambda}}$ of system (12), (13) have the following forms:

$$
\begin{aligned}
& A_{11_{\lambda}}=\left[\begin{array}{cccc}
A_{11_{\lambda}}^{*} & 0 & & 0 \\
A_{21_{\lambda}}^{*} & A_{22_{\lambda}}^{*} & & 0 \\
\vdots & & \ddots & \\
A_{n^{*} 1_{\lambda}}^{*} & A_{n^{*} 1_{\lambda}}^{*} & \cdots & A_{p^{*} p_{\lambda}^{*}}^{*}
\end{array}\right] \\
& A_{i i_{\lambda}}^{*}=\left[\begin{array}{ccccc}
0 & 1 & 0 & \cdots & 0 \\
0 & 0 & 1 & & 0 \\
\vdots & & & \ddots & \vdots \\
0 & 0 & 0 & & 1 \\
& * & * & \cdots & *
\end{array}\right] \\
& A_{i j_{\lambda}}^{*}=\left[\begin{array}{ccc}
0 & \cdots & 0 \\
\vdots & & \\
0 & \cdots & 0 \\
* & \cdots & *
\end{array}\right], D_{1_{\lambda}}=\left[\begin{array}{c}
D_{1_{\lambda}}^{*} \\
\vdots \\
D_{n_{\lambda}^{*}}
\end{array}\right], E_{1_{\lambda}}=\left[\begin{array}{c}
E_{1_{\lambda}}^{*} \\
\vdots \\
E_{n_{\lambda}^{*}}
\end{array}\right], \\
& D_{j_{\lambda}}^{*}=\left[\begin{array}{ccc}
0 & \cdots & 0 \\
\vdots & & \\
0 & \cdots & 0 \\
* & \cdots & *
\end{array}\right], E_{i_{\lambda}}^{*}=\left[\begin{array}{ccc}
0 & \cdots & 0 \\
\vdots & & \\
0 & \cdots & 0 \\
* & \cdots & *
\end{array}\right] \\
& \left.C_{11_{\lambda}}=\left[\begin{array}{ccccccccc}
1 & 0 & \cdots & 0 & & & 0 & & \\
& & & 1 & 0 & \cdots & 0 & & \\
& & & & \ddots & & & & \\
& & & & & & 1 & \cdots & 0
\end{array}\right]\right\} q_{1}
\end{aligned}
$$

where $*$ denotes a matrix block of no interest in the present discussion.

\section{B. Observer design}

System (12), (13) has a block structure form that allows us to design an observer according to the characteristics of each state.
State $\chi_{1}$ corresponds to the set of states that are reconstructible (in finite-time) despite the disturbances. The dynamics of $\chi_{1}$ can be written as follows

$$
\begin{aligned}
&{ }^{i} \dot{\chi}_{1}= \sum_{j=1}^{i-1} A_{1 j_{\lambda}}^{*}\left({ }^{j} \chi_{1}\right)+A_{i i_{\lambda}}^{*}\left({ }^{i} \chi_{1}\right)+B_{1 i_{\lambda}} u+ \\
& y_{1_{i}}={ }^{i} \chi_{1 i_{\lambda}} d(t)+E_{1 i_{\lambda}}^{*} w(t)
\end{aligned}
$$

each one of the $q_{1}$ blocks (14) could be written as:

$$
\begin{aligned}
{ }^{i} \dot{\chi}_{1_{j}} & ={ }^{i} \chi_{1_{(j+1)}}+B_{1 i_{(j)_{\lambda}}}^{*} u, j=\overline{1, r_{i}-1} \\
{ }^{i} \dot{\chi}_{1_{\left(r_{i}\right)}} & ={ }{ }_{i_{\lambda}} A_{\lambda}^{r_{i}} x+B_{1 i_{\left(r_{i}\right)_{\lambda}}^{*}}^{*} u+D_{1 i_{\left(r_{i}\right)_{\lambda}}}^{*} d(t)+E_{1 i_{\left(r_{i}\right)_{\lambda}}^{*}} w(t)
\end{aligned}
$$

where ${ }^{i} \chi_{1_{j}}, j=\overline{1, r_{i}-1}$ denotes the $j$-th element of the partitioned state vector $\chi_{1}$ corresponding to the block $i, i=\overline{1, q_{1}}$.

An estimated of the variable $\chi_{1}$ can be obtained from the auxiliary variable $\hat{z}_{1}$ and the following nonlinear equation:

$$
\begin{aligned}
{ }^{i} \dot{\hat{z}}_{1_{j}}= & B_{1_{(j)_{\lambda}}}^{*} u-k_{j}(L(t))^{\frac{j}{r_{i}}}\left\lceil{ }^{i} \hat{z}_{1_{1}}-{ }^{i} \chi_{1_{1}}\right]^{\frac{r_{i}-j}{r_{i}}}+ \\
& { }^{i} \hat{z}_{1_{(j+1)}}, j=\overline{1, r_{i}-1} \\
{ }^{i} \dot{\hat{z}}_{1_{\left(r_{i}\right)}}= & B_{1_{\left(r_{i}\right)_{\lambda}}^{*}}^{*} u-k_{r_{i}} L(t)\left\lceil{ }^{i} \hat{z}_{1_{1}}-{ }^{i} \chi_{1_{1}}\right\rfloor^{0}
\end{aligned}
$$

where $L(t)$ is a time-varying gain that satisfies for a chosen $\varepsilon \in \mathbb{R}^{+}$the equality $L(t)=\|C\|\|\mid A\| \bar{x}+\bar{d}(t)+\bar{E} \bar{w}+\varepsilon$. To estimate the remaining state variable, which is affected by the unknown inputs, let introduce the auxiliary variable $\hat{z}_{2}$. Then, a collaborative estimation is obtained as follows:

$$
\begin{aligned}
\dot{z}_{2}^{+}= & G_{2_{\lambda}} A_{21_{\lambda}} \hat{z}_{1}+\left(G_{2_{\lambda}} A_{22_{\lambda}} G_{2_{\lambda}}^{-1}\right) \hat{z}_{2}^{+}+G_{2_{\lambda}} B_{2_{\lambda}} u(t) \\
& +G_{2_{\lambda}} L_{2_{\lambda}}\left(y_{2}-C_{22_{\lambda}} G_{2_{\lambda}}^{-1} \hat{z}_{2}^{+}\right) \\
& +\left(\left[\left(G_{2_{\lambda}}\right)^{+}+\left(G_{2_{\lambda}}\right)^{-}\right] 1 \bar{E} \bar{w}\right)
\end{aligned}
$$

and

$$
\begin{aligned}
\dot{\hat{z}}_{2}^{-}= & G_{2_{\lambda}} A_{21_{\lambda}} \hat{z}_{1}+\left(G_{2_{\lambda}} A_{22_{\lambda}} G_{2_{\lambda}}^{-1}\right) \hat{z}_{2}^{-}+G_{2_{\lambda}} B_{2_{\lambda}} u(t) \\
& +G_{2_{\lambda}} L_{2_{\lambda}}\left(y_{2}-C_{22_{\lambda}} G_{2_{\lambda}}^{-1} \hat{z}_{2}^{-}\right) \\
& -\left(\left[\left(G_{2_{\lambda}}\right)^{+}+\left(G_{2_{\lambda}}\right)^{-}\right] 1 \bar{E} \bar{w}\right) .
\end{aligned}
$$

where $1 \in \mathbb{R}^{\left(n-n \mathcal{V}_{\lambda}-n_{\left.\mathcal{N}_{\lambda}\right) \times 1}\right.}$ is a vector of ones, and $L_{2_{\lambda}}$ is chosen such that, under Assumption 2, there exists a matrix $G_{2_{\lambda}}$ for which $\tilde{A}_{\lambda}=G_{2_{\lambda}}\left(A_{22_{\lambda}}-L_{2 \lambda} C_{22_{\lambda}}\right) G_{2_{\lambda}}^{-1}$ is a Metzler matrix that satisfies the following inequality

$$
P_{\lambda} \tilde{A}_{\lambda}+\tilde{A}_{\lambda}^{T} P_{\lambda}+\frac{1}{\delta} P_{\lambda}<-J_{\lambda}
$$

for symmetric positive definite matrices $P_{\lambda}, J_{\lambda} \in$ $\mathbb{R}^{\left(n-n_{\mathcal{V}_{\lambda}}\right) \times\left(n-n \mathcal{V}_{\lambda}\right)}$ and a positive scalar $\delta>0$. For a procedure to compute the matrix $G_{2_{\lambda}}$ please refer to [26].

Let define the following vectors:

$$
\hat{z}^{+}=\left[\begin{array}{c}
\hat{z}_{1} \\
\hat{z}_{2}^{+}
\end{array}\right], \quad \hat{z}^{-}=\left[\begin{array}{c}
\hat{z}_{1} \\
\hat{z}_{2}^{-}
\end{array}\right],
$$

and the transformation matrix:

$$
T_{\lambda}=\left[\begin{array}{c}
O_{\lambda} \\
G_{2_{\lambda}} V_{\lambda}
\end{array}\right]
$$

The following reset equations are proposed for each switching time $t_{i}$ 


$$
\begin{aligned}
& \hat{z}^{+}\left(t_{i}^{+}\right)=\left[\begin{array}{c}
O_{i+1} \\
G_{2_{i+1}} V_{i+1}
\end{array}\right]\left[\begin{array}{c}
O_{i} \\
G_{2_{i}} V_{i}
\end{array}\right]^{-1} \hat{z}^{+}\left(t_{i}^{-}\right) \\
& \hat{z}^{-}\left(t_{i}^{+}\right)=\left[\begin{array}{c}
O_{i+1} \\
G_{2_{i+1}} V_{i+1}
\end{array}\right]\left[\begin{array}{c}
O_{i} \\
G_{2_{i}} V_{i}
\end{array}\right]^{-1} \hat{z}^{-}\left(t_{i}^{-}\right)
\end{aligned}
$$

where $t_{i}^{-}$is the time instant just before the switching time $t_{i}$, and $t_{i}^{+}$is the time instant just after the switching time $t_{i}$.

Define the estimation error $e_{1}=z_{1}-\hat{z}_{1}$. By following the procedure described in [27], a Lyapunov function for each block of the system (12), (16) is given by:

$$
{ }^{i} V_{\lambda}=\sum_{j=1}^{r_{i}-1} \beta_{j}{ }^{i} \gamma_{j}\left({ }^{i} e_{1_{j}},{ }^{i} e_{1_{j+1}}\right)+\beta_{n} \frac{1}{p_{i}}\left|{ }^{i} e_{1_{r_{i}}}\right|^{p},
$$

where

$$
\begin{aligned}
& { }^{i} \gamma_{j}\left({ }^{i} e_{1_{j}},{ }^{i} e_{1_{j+1}}\right)=\frac{r_{i}+1-j}{p_{i}}\left|{ }^{i} e_{1_{j}}\right|^{\frac{p_{i}}{r_{i}+1-j}}+ \\
& -{ }^{i} e_{1_{j}}\left\lceil{ }^{i} e_{1_{j+1}}\right\rfloor^{\frac{p_{i}-r_{i}-1+j}{r_{i}-j}}+ \\
& \left.\left.\left(\frac{p_{i}-r_{i}-1+j}{p_{i}}\right)\right|^{i} e_{1_{j+1}}\right|^{\frac{p_{i}}{r_{i}-j}}
\end{aligned}
$$

and $\beta_{k}>0, k=\overline{1, r_{i}}, p_{i}=2 r_{i}-1$ and $\beta_{i}>0$. The setup time for each block is computed as

$$
t_{\text {setup }, i_{\lambda}}(\max (|\bar{x}|,|\underline{x}|))=\left(\frac{p_{i}}{\kappa_{1}}\left({ }^{i} V_{\lambda}{ }^{i}\left(\left(\overline{{ }^{i} e_{1}\left(t_{i}\right)}\right)\right)\right)^{\frac{1}{p_{i}}}\right)
$$

where $\overline{{ }^{\lambda} e_{1}}=\max (|\bar{x}|,|\underline{x}|), \max (|\bar{x}|,|\underline{x}|)$ is the maximal value between upper and lower bounds (3). ${ }^{i} e_{1}\left(t_{i}\right)={ }^{i}$ $z_{1}-{ }^{i} \hat{z}_{1}$ are the components of the $i$ th block of $z_{1}-\hat{z}_{1}$, respectively. Therefore, there exists a maximal setup time:

$$
t_{\text {setup, } \max _{\lambda}}(\max (|\bar{x}|,|\underline{x}|))=\max _{i=\overline{1, N}}\left\{t_{\text {setup }, i_{\lambda}}(\max (|\bar{x}|,|\underline{x}|))\right\}
$$

Assumption 4: There exists a positive constant $\Upsilon_{\delta}>0$ called the dwell-time, such that the time between two consecutive switchings of the system (1), (2) satisfy $\left(t_{(i+1)}-t_{i}\right) \leq$ $\Upsilon_{\delta}, i \in \mathbb{N}$,

$$
\begin{aligned}
& \Upsilon_{\delta}=\max _{\lambda=\overline{1, N}}\left\{t_{\text {setup }, \max _{\lambda}}\left(T_{\lambda}(\bar{x}-\underline{x})\right)\right. \\
& \left.-2 \frac{\lambda_{\max }\left(P_{\lambda}\right)}{\lambda_{\min }\left(J_{\lambda}\right)} \ln \left(\frac{\lambda_{\min }\left(P_{\lambda}\right)}{\lambda_{\max }\left(P_{\lambda}\right)}\right)\right\}
\end{aligned}
$$

Theorem 1: Let the system (1), (2) satisfy Assumptions $1,2,3$ and 4, then the collaborative estimated

$$
\begin{aligned}
& \underline{\hat{x}}=\left(T_{\lambda}^{-1}\right)^{+} \hat{z}^{-}-\left(T_{\lambda}^{-1}\right)^{-} \hat{z}^{+} \\
& \overline{\hat{x}}=\left(T_{\lambda}^{-1}\right)^{+} \hat{z}^{+}-\left(T_{\lambda}^{-1}\right)^{-} \hat{z}^{-}
\end{aligned}
$$

with the auxiliary variables (16), (17), (18), (19) provides, after a dwell-time $\Upsilon_{\delta}$, an interval estimation of the state in spite of the presence of $d(t)$ and $w(t)$.

Proof: Let represent the transformation matrix $T_{\lambda}$ as the product of two matrices:

$$
T_{\lambda}=G_{\lambda} M_{\lambda}, \quad G_{\lambda}=\left[\begin{array}{cc}
I & 0 \\
0 & G_{2_{\lambda}}
\end{array}\right]
$$

Thus, the similarity transformation $z=T_{\lambda} x$ could be seen as the result of the successive transformations $z=G_{\lambda} \chi$ and $\chi=M_{\lambda} x$. The first transformation, which is a decoupling transformation, brings the state of the system to the form (12). Transformation $y_{z}=S_{\lambda} y$ is introduced to decouple and reorder the outputs to obtain the normal form (13).

The dynamics of the state in the new coordinates $z$ is:

$$
\begin{aligned}
{\left[\begin{array}{c}
\dot{z}_{1} \\
\dot{z}_{2}
\end{array}\right]=} & {\left[\begin{array}{cc}
A_{11_{\lambda}} & 0 \\
G_{2_{\lambda}} A_{21_{\lambda}} & \left(G_{2_{\lambda}} A_{22_{\lambda}} G_{2_{\lambda}}^{-1}\right)
\end{array}\right]\left[\begin{array}{l}
z_{1} \\
z_{2}
\end{array}\right] } \\
& +\left[\begin{array}{c}
B_{1_{\lambda}} \\
G_{2_{\lambda}} B_{2_{\lambda}}
\end{array}\right] u(t)+\left[\begin{array}{c}
D_{1_{\lambda}} \\
0
\end{array}\right] d(t) \\
& +\left[\begin{array}{c}
E_{1_{\lambda}} \\
G_{2_{\lambda}} E_{2_{\lambda}}
\end{array}\right] w(t) \\
{\left[\begin{array}{c}
y_{z_{1}} \\
y_{z_{2}}
\end{array}\right]=} & {\left[\begin{array}{cc}
C_{11_{\lambda}} & 0 \\
0 & \left(C_{22_{\lambda}} G_{2_{\lambda}}^{-1}\right)
\end{array}\right]\left[\begin{array}{c}
z_{1} \\
z_{2}
\end{array}\right] }
\end{aligned}
$$

Recall that $z_{1}=\chi_{1}$, the observer (16) is a point observer which takes advantage of the full relative degree feature to guarantee the finite time convergence of the estimation error $e_{1}=\hat{z}_{1}-z_{1}$. The estimation error can be studied in the block form (14), (15) as:

$$
\begin{aligned}
{ }^{i} \dot{e}_{1_{j}} & ={ }^{i} e_{1_{j+1}}-k_{j}(L(t))^{j / r_{i}}\left\lceil{ }^{i} e_{1_{1}}\right\rfloor^{\left(r_{i-j} / r_{i}\right)} \\
{ }^{i} \dot{e}_{1_{r_{i}}} & =-\left(c_{i_{\lambda}} A_{\lambda}^{r_{i}} x+D_{i_{r_{i}}}^{*} d(t)+E_{i_{r_{i}}}^{*} w(t)\right)-k_{r_{i}} L(t)\left\lceil{ }^{i} e_{1_{1}}\right\rfloor^{0}
\end{aligned}
$$

It is important to remark that each operation mode $\lambda$ may have a different block structure. In that case, it may require a proper design of the transformations and observer matrices, as well as a careful selection of the initial conditions. Introducing the change of variables ${ }^{i} \xi_{1_{1}}=\frac{{ }^{i} e_{1_{1}}}{L(t)},{ }^{i} \xi_{1_{2}}=$ $\frac{{ }^{i} e_{1_{2}}}{k_{1} L(t)}, \ldots,{ }^{i} \xi_{1_{j}}=\frac{{ }^{i} e_{1_{j}}}{k_{(j-1)} L(t)}, \quad j=\overline{1, r_{i}}$.

Then, the error dynamics in the new variables is given by

$$
\begin{aligned}
{ }^{i} \dot{\xi}_{1_{1}}= & k_{1}\left({ }^{i} \xi_{1_{2}}-\left\lceil{ }^{i} \xi_{1_{1}}\right\rfloor^{\frac{r_{i}-1}{r_{i}}}\right)-\frac{\dot{L}(t)}{L(t)} i_{1_{1}} \\
{ }^{i} \dot{\xi}_{1_{j}}= & \frac{k_{j}}{k_{j-1}}\left({ }^{i} \xi_{1_{j+1}}-\left\lceil{ }^{i} \xi_{1_{1}}\right\rfloor^{\frac{r_{i}-j}{r_{i}}}\right)-\frac{\dot{L}(t)}{L(t)}{ }^{i} \xi_{1_{j}} \\
{ }^{i} \xi_{1_{r_{i}}}= & -\frac{k_{r_{i}}}{k_{r_{i}-1}}\left(\frac{1}{k_{r_{i}}}\left(c_{i} A^{r_{i}} x+D_{i_{r_{i}}}^{*} d(t)+E_{i_{r_{i}}}^{*} w(t)\right)\right. \\
& \left.+\left\lceil{ }^{i} \xi_{1_{1}}\right\rfloor^{0}\right)-\frac{\dot{L}(t)}{L(t)}{ }^{i} \xi_{1_{r_{i}}}
\end{aligned}
$$

for $j=\overline{2,\left(r_{i}-1\right)}$. Let now follow the procedure presented in [27] to prove that all ${ }^{i} \xi_{1}, i=\overline{1, q_{1}}$ converge to zero in finite time. Consider again the Lyapunov candidate functions (21), and let us rewrite the function as:

$$
{ }^{i} V_{\lambda}=\sum_{j=1}^{r_{i}-1} \beta_{j}{ }^{i} \gamma_{j}\left({ }^{i} \xi_{1_{j}},{ }^{i} \xi_{1_{j+1}}\right)+\beta_{n} \frac{1}{p}\left|{ }^{i} \xi_{1_{r_{i}}}\right| p, \quad \beta_{k}>0,
$$

with $k=\overline{1, r_{i}}$ and

$$
\begin{aligned}
{ }^{i} \gamma_{j}\left({ }^{i} \xi_{1_{j}},{ }^{i} \xi_{1_{j+1}}\right)= & \frac{r_{i}+1-j}{p}\left|{ }^{i} \xi_{1_{j}}\right|{ }^{\frac{p}{r_{i}+1-j}} \\
& -\left.\left.{ }^{i} \xi_{1_{j}}\right|^{i} \xi_{1_{j+1}}\right|^{\frac{p-r_{i}-1+j}{r_{i}-j}} \\
& +\left(\frac{p-r_{i}-1+j}{p}\right)\left|{ }^{i} \xi_{1_{j+1}}\right|^{\frac{p}{r_{i}-j}}
\end{aligned}
$$


where $p=2 \max _{\forall i}\left(r_{i}\right)-1$ and $\beta_{i}>0$. Therefore, it was proven in [27], that there exist constants $\kappa_{1}$ and $\kappa_{2}$ such that the derivative of the Lyapunov candidate (26) satisfies:

$$
{ }^{i} \dot{V}_{\lambda} \leq-\kappa_{1}\left({ }^{i} V_{\lambda}\right)^{\frac{p-1}{p}}-\frac{\dot{L}(t)}{L(t)} \kappa_{2}{ }^{i} V_{\lambda}
$$

in particular, $\kappa_{2}$ is a function, $\kappa_{2}(\dot{L}(t))$.

The above given inequality allows to guarantee the finite time convergence to zero of ${ }^{i} \xi_{1}$, with a setup time given by the following expression:

$$
\Upsilon\left({ }^{i} \xi_{1}\left(t_{i}^{+}\right)\right) \leq \frac{p}{\kappa_{1}}\left({ }^{i} V_{\lambda}\left({ }^{i} \xi_{1}\left(t_{i}^{+}\right)\right)\right)^{\frac{1}{p}}
$$

where $t_{i}^{+}$is the $i$ th switching time.

Therefore, there exists a maximal setup time that can be represented as

$$
\Upsilon_{\max }\left(\xi_{1}\left(t_{i}^{+}\right)\right) \leq \max _{i=\overline{1, q_{1}}}\left(\frac{p}{\kappa_{1}}\left({ }^{i} V_{\lambda}\left({ }^{i} \xi_{1}\left(t_{i}^{+}\right)\right)\right)^{\frac{1}{p}}\right)
$$

Notice that, in terms of the upper bounds of the states given in (3), a maximal setup time can be computed as (22).

The interval estimation error for the state $z_{2}$ is defined by the couple of variables $e_{2}^{+}=\hat{z}_{2}^{+}-z_{2}, \quad e_{2}^{-}=z_{2}-\hat{z}_{2}^{-}$. Thus, their dynamics are presented in the following equations:

$$
\begin{aligned}
& \dot{e}_{2}^{+}=H_{1} e_{1}+\tilde{A}_{\lambda} e_{2}^{+}+\gamma_{w}^{+} \\
& \dot{e}_{2}^{-}=H_{1} e_{1}+\tilde{A}_{\lambda} e_{2}^{-}+\gamma_{w}^{-}
\end{aligned}
$$

where $\tilde{A}_{\lambda}=G_{2_{\lambda}}\left(A_{22_{\lambda}}-L_{2 \lambda} C_{22_{\lambda}}\right) G_{2_{\lambda}}^{-1}, H_{1}=$ $G_{2_{\lambda}} A_{21_{\lambda}}, \gamma_{w}^{+}=\left[\left(G_{2_{\lambda}}\right)^{+}+\left(G_{2_{\lambda}}\right)^{-}\right] 1 \bar{E} \bar{w}$, and $\gamma_{w}^{-}=$ $-\left[\left(G_{2_{\lambda}}\right)^{+}+\left(G_{2_{\lambda}}\right)^{-}\right] 1 \bar{E} \bar{w}$.

Thus, three elements compose the estimation error (29) and (30). A non-collaborative component that depends on $e_{1}$ which vanishes after a finite-time transient given by (27); and a second term that is integrated by the error variables $e_{2}^{+}$and $e_{2}^{-}$, that under the satisfaction of the inequality (20) is ruled by a Metzler matrix, which provides for a positive dynamics; finally, a third term composed of the unknown input information $\gamma_{w}^{+}, \gamma_{w}^{-}$, which is, by design, a strictly positive term, the rest of the proof is a consequence of the work by [28].

A Lyapunov candidate function to prove the stability of the estimation error is given by

$$
V_{\lambda}^{+}=\sum_{i=1}^{q_{1}}{ }^{i} V_{\lambda}+\tilde{e}^{+T} P_{\lambda} \tilde{e}^{+}
$$

Computing the time derivative of (31):

$$
\dot{V}_{\lambda}^{+}=\sum_{i=1}^{q_{1}}{ }^{i} \dot{V}_{\lambda}+\tilde{e}^{+T} P_{\lambda} \dot{\tilde{e}}^{+}+\dot{\tilde{e}}^{+T} P_{\lambda} \tilde{e}^{+}
$$

Substituting (29) into (32), the following equality is obtained

$$
\begin{aligned}
\dot{V}_{\lambda}^{+}= & \sum_{i=1}^{q_{1}}{ }^{i} \dot{V}_{\lambda}+\tilde{e}^{+T} P_{\lambda}\left(H_{1} e_{1}+\tilde{A}_{\lambda} \tilde{e}^{+}+\gamma_{w}^{+}\right) \\
& +\left(H_{1} e_{1}+\tilde{A}_{\lambda} \tilde{e}^{+}+\gamma_{w}^{+}\right)^{T} P_{\lambda} \tilde{e}^{+}
\end{aligned}
$$

Recall that after a finite time transient $\Upsilon_{\max }\left(\xi_{1}\left(t_{i}^{+}\right)\right)$, the estimation error $e_{1}$ vanishes and its contribution to the Lyapunov function is null, therefore for all $t \geq \Upsilon_{\max }\left(\xi_{1}\left(t_{i}^{+}\right)\right)$ the following equality holds

$$
\dot{V}_{\lambda}^{+}=\tilde{e}^{+T}\left(P_{\lambda} \tilde{A}_{\lambda}+\tilde{A}_{\lambda}^{T} P_{\lambda}\right) \tilde{e}^{+}+2 \tilde{e}^{+T} P_{\lambda} \gamma_{w}^{+}
$$

Applying Lemma 1 to the last term of the equality:

$\dot{V}_{\lambda}^{+} \leq \tilde{e}^{+T}\left(P_{\lambda} \tilde{A}_{\lambda}+\tilde{A}_{\lambda}^{T} P_{\lambda}+\frac{1}{\delta} P_{\lambda}\right) \tilde{e}^{+}+\delta \gamma_{w}^{+T} P_{\lambda} \gamma_{w}^{+}$

$\dot{V}_{\lambda}^{+} \leq-\tilde{e}^{+T} J_{\lambda} \tilde{e}^{+}+\delta \gamma_{w}^{+T} P_{\lambda} \gamma_{w}^{+}$

Notice that the last term in the right-hand side of (33) satisfies the following inequality $\left\|\gamma_{w}^{+T} P_{\lambda} \gamma_{w}^{+}\right\| \leq$ $\left\|P_{\lambda}\right\|\left\|G_{2_{\lambda}} E_{2_{\lambda}}\right\|^{2} \bar{w}^{2}$. It is clear that all the terms in the righthand side of the inequality are bounded, therefore under a proper selection of the gains $L_{2_{\lambda}}$ and $L_{3_{\lambda}}$, and given the existence of a matrix $G_{2_{\lambda}}$ such that the inequality (20) is satisfied, the equation (29) is Input to State Stable, and the estimation error $\tilde{e}^{+}$is bounded.

In a similar way, using the following Lyapunov candidate:

$$
V_{\lambda}^{-}=\sum_{i=1}^{q_{1}}{ }^{i} V_{\lambda}+\tilde{e}^{-T} P_{\lambda} \tilde{e}^{-}
$$

whose derivative satisfies

$$
\dot{V}_{\lambda}^{-} \leq \tilde{e}^{-T}\left(P_{\lambda} \tilde{A}_{\lambda}+\tilde{A}_{\lambda}^{T} P_{\lambda}+\frac{1}{\delta} P_{\lambda}\right) \tilde{e}^{-}+\delta \gamma_{w}^{-T} P_{\lambda} \gamma_{w}^{-}
$$

The uncertain term in the right-hand side satisfies the norm inequality $\left\|\gamma_{w}^{-T} P_{\lambda} \gamma_{w}^{-}\right\| \leq\left\|P_{\lambda}\right\|\left\|G_{2_{\lambda}} E_{2_{\lambda}}\right\|^{2} \bar{w}^{2}$. Notice that, after the dwell-time given in equation (28), the estimation error $\tilde{e}^{-}$is bounded.

After a time transient of duration $\Upsilon_{\max }$, the estimated values $\hat{z}^{+}$and $\hat{z}^{-}$provide an upper and lower estimation of the state $z$. Therefore, the collaborativeness of the estimated states (24), (25) is a direct consequence of the application of Lemma 1.

After the setup time (28), the following Lyapunov candidate can be used to prove the convergence

$$
V_{\lambda, \Upsilon_{\max }}^{+}=\tilde{e}^{+T} P_{\lambda} \tilde{e}^{+}
$$

Due that (34) has a quadratic form, then it satisfy

$$
\lambda_{\min }\left(P_{\lambda}\right)\left\|\tilde{e}^{+}(t)\right\|^{2} \leq V_{\lambda, \Upsilon_{\max }}(t) \leq \lambda_{\max }\left(P_{\lambda}\right) \tilde{e}^{+T} \tilde{e}^{+}
$$

Provided the setup-time $\Upsilon_{\max }$, the linear part of the observer governs the convergence of the states. Then, for all $t \geq \Upsilon_{\max }$ the derivative of (31) satisfies:

$$
\begin{aligned}
\dot{V}_{\lambda, \Upsilon_{\max }}^{+} \leq & -\tilde{e}^{+T} J_{\lambda} \tilde{e}^{+}+\delta \gamma_{w}^{+T} P_{\lambda} \gamma_{w}^{+} \\
\leq & -\lambda_{\min }\left(J_{\lambda}\right) \tilde{e}^{+T} \tilde{e}^{+}+\left\|\delta \gamma_{w}^{+T} P_{\lambda} \gamma_{w}^{+}\right\| \\
\leq & -\frac{\lambda_{\min }\left(J_{\lambda}\right)}{\lambda_{\max }\left(P_{\lambda}\right)} V_{\lambda, \Upsilon_{\max }}^{+}+\left\|\delta \gamma_{w}^{+T} P_{\lambda} \gamma_{w}^{+}\right\|
\end{aligned}
$$

The Lyapunov function (31) verifies $V_{\lambda, \Upsilon_{\max }}^{+}(t) \leq$ $e^{-\alpha t} V_{\lambda, \Upsilon_{\max }}\left(t_{i}^{+}\right)$, where $t_{i}^{+}$is the switching time and $\alpha=$ 
$\frac{\lambda_{\min }\left(J_{\lambda}\right)}{\lambda_{\max }\left(P_{\lambda}\right)}$. Due that the inequality (35) is satisfied, the following chain of inequalities are also satisfied

$$
\lambda_{\min }\left(P_{\lambda}\right)\left\|\tilde{e}^{+}(t)\right\|^{2} \leq V_{\lambda, \Upsilon_{\max }}^{+}(t) \leq \lambda_{\max }\left(P_{\lambda}\right) e^{-\alpha t}\left\|e^{+}\left(t_{i}^{+}\right)\right\|^{2}
$$

Therefore the following inequality is also satisfied:

$$
\left\|\tilde{e}^{+}(t)\right\| \leq \sqrt{\frac{\lambda_{\max }\left(P_{\lambda}\right)}{\lambda_{\min }\left(P_{\lambda}\right)}} e^{-\frac{\alpha t}{2}}\left\|e^{+}\left(t_{i}^{+}\right)\right\|
$$

In order to guarantee the convergence of the estimation error, the next inequality must be satisfied

$$
\sqrt{\frac{\lambda_{\max }\left(P_{\lambda}\right)}{\lambda_{\min }\left(P_{\lambda}\right)}} e^{-\frac{\alpha t}{2}}<1
$$

Then, the following dwell-time is obtained for the collaborative part of the observer:

$$
\Upsilon_{\delta}>\Upsilon_{\max }\left(\xi_{1}(0)\right)-\frac{2}{\alpha} \ln \left(\frac{\lambda_{\min }\left(P_{\lambda}\right)}{\lambda_{\max }\left(P_{\lambda}\right)}\right)
$$

Therefore, the algorithm guarantees the exponential convergence of the collaborative estimates as long as the switching times satisfy dwell-time (23), as given in Assumption 4.

Finally, applying Lemma 2 it is possible to design a collaborative estimation of the original state as it was presented in (24) and (25); then, the theorem has been proven.

\section{Conclusions}

In this article, a collaborative estimation technique is proposed for a class of Linear Switched Systems affected by two classes of disturbances. To solve the problem, two estimation algorithms are used. A point observer that reconstruct the states affected by disturbances with a time-varying upper bound, and a collaborative observer that estimates a part of the state, which relative degree is not well known, and is affected by bounded unknown inputs.

\section{REFERENCES}

[1] B. Hu, G. Zhai, and A. N. Michel, "Common quadratic lyapunov-like functions with associated switching regions for two unstable secondorder lti systems," International Journal of Control, vol. 75, no. 14, pp. 1127-1135, 2002.

[2] U. Serres, J. Vivalda, and P. Riedinger, "On the convergence of linear switched systems," IEEE Transactions on Automatic Control, vol. 56, no. 2, pp. 320-332, 2011.

[3] M. Baglietto, G. Battistelli, and L. Scardovi, "Active mode observation of switching system based on a-priori knowledge of the continuous state," in Proc. 46th IEEE Conf. Decision Control, New Orleans, USA, 2007, pp. 1752-1757.

[4] A. Alessandri and P. Coletta, "Design of observers for switched discrete-time linear systems," in Proc. 2003 American Control Conference, Denver, CO, USA, 2003, pp. 2785-2790.

[5] F. Bejarano, A. Pisano, and E. Usai, "Finite-time converging jump observer for switched linear systems with unknown inputs," Nonlinear Analysis: Hybrid Systems, vol. 5, no. 2, pp. 174-188, 2011.

[8] F. Bejarano and L. Fridman, "State exact reconstruction for switched linear systems via a super-twisting algorithm," International Journal of Systems Science, vol. 42, no. 5, pp. 717-724, 2011.
[6] L. Pina and M. Botto, "Simultaneous state and input estimation of hybrid systems with unknown inputs," Automatica, vol. 42, no. 5, pp. 1755-1762, 2006.

[7] N. Orani, A. Pisano, M. Franceschelli, A. Giua, and E. Usai, "Robust reconstruction of the discrete state for a class of nonlinear uncertain switched systems," Nonlinear Analysis: Hybrid Systems, vol. 5, no. 2, pp. 220-232, 2011.

[9] L. Vu and D. Liberzon, "Invertibility of switched linear systems," Automatica, vol. 44, no. 4, pp. 949-958, 2008.

[10] M. Defoort, M. Djemai, T. Floquet, and W. Perruquetti, "Robust finite time observer design for multicellular converters," Int. J. Systems Science, vol. 42, no. 11, pp. 1859-1868, 2011.

[11] J. Davila, H. Ríos, and L. Fridman, "State observation for nonlinear switched systems using nonhomogeneous high-order sliding mode observers," Asian Journal of Control, vol. 14, no. 4, pp. 911-923, 2012.

[12] H. Ríos, J. Davila, and L. Fridman, "High-order sliding mode observers for nonlinear autonomous switched systems with unknown inputs," Journal of the Franklin Institute, vol. 349, no. 10, pp. 2975 3002, 2012.

[13] F. Mazenc, T. N. Dinh, and S. I. Niculescu, "Interval observers for discrete-time systems," International Journal of Robust and Nonlinear Control, vol. 24, no. 17, pp. 2867-2890, 2014.

[14] R. Lamouchi, M. Amairi, T. Raissi, and M. Aoun, "Interval observer design for linear parameter-varying systems subject to component faults," in 2016 24th Mediterranean Conference on Control and Automation (MED), 2016, pp. 707-712.

[15] D. C. Huong, V. T. Huynh, and H. Trinh, "Interval functional observers design for time-delay systems under stealthy attacks," IEEE Transactions on Circuits and Systems I: Regular Papers, vol. 67, no. 12, pp. 5101-5112, 2020.

[16] — "Distributed functional interval observers for nonlinear interconnected systems with time-delays and additive disturbances," IEEE Systems Journal, vol. 15, no. 1, pp. 411-422, 2021.

[17] C. M. Nguyen, C. P. Tan, and H. Trinh, "Sliding mode observer for estimating states and faults of linear time-delay systems with outputs subject to delays," Automatica, vol. 124, p. 109274, 2021.

[18] D. Efimov, L. Fridman, T. Raïssi, A. Zolghadri, and R. Seydou, "Interval estimation for LPV systems applying high order sliding mode techniques," Automatica, vol. 48, no. 9, pp. 2365 - 2371, 2012.

[19] D. Gucik-Derigny, T. Raïssi, and A. Zolghadri, "A note on interval observer design for unknown input estimation," International Journal of Control, vol. 89, no. 1, pp. 25-37, 2016.

[20] H. Ethabet, T. Raïssi, M. Amairi, C. Combastel, and M. Aoun, "Interval observer design for continuous-time switched systems under known switching and unknown inputs," International Journal of Control, vol. 0, no. 0, pp. 1-14, 2018.

[21] B. P. Molinari, "A strong controllability and observability in linear multivariable control," IEEE Trans. Automat. Contr., vol. 21, no. 5, pp. 761-764, 1976.

[22] G. Hardy, J. Littlewood, and G. Pólya, Inequalities. London: Cambridge University Press, 1953.

[23] H. Minc, Nonnegative Matrices, ser. Wiley Series in Discrete Mathematics and Optimization. USA: Wiley-Interscience, 1988.

[24] L. Farina and S. Rinaldi, Positive Linear Systems: Theory and Applications, ser. Pure and Applied Mathematics. USA: Wiley-Interscience, 2000.

[25] L. Fridman, A. Levant, and J. Davila, "Observation of linear systems with unknown inputs via high-order sliding-modes," Int. J. System Science, vol. 38, no. 10, pp. 773-791, 2007.

[26] T. Raïssi, D. Efimov, and A. Zolghadri, "Interval state estimation for a class of nonlinear systems," IEEE Transactions on Automatic Control, vol. 57, no. 1, pp. 260-265, Jan 2012.

[27] J. A. Moreno, "Exact differentiator with varying gains," International Journal of Control, vol. 91, no. 9, pp. 1983-1993, 2018.

[28] J. Gouzé, A. Rapaport, and M. Hadj-Sadok, "Interval observers for uncertain biological systems," Ecological Modelling, vol. 133, no. 1, pp. $45-56,2000$. 